\title{
L'électrophorèse capillaire en milieu non aqueux
}

\author{
A. Desbène-Monvernay et N. Mofaddel
}

\section{Laboratoire d'Analyse des Systèmes Organiques Complexes, IRCOF-UPRES EA 2659 et IFRMP, 43 rue Saint-Germain, 27000 Evreux,} France

\begin{abstract}
The utilisation of Capillary Electrophoresis in hydroorganic and organic media is gradually increasing since fifteen years. The influence of the physicochemical properties of ten organic solvents on the fundamental parameters commanding an electrophoretic separation, i.e. current efficiency, analysis time, resolution, is considered. The use of non aqueous media offers new attractive possibilities for the analysis of hydrophobic compounds by Capillary Electrophoresis. The choice of convenient solvent allows an easier optimisation of selectivity, particularly in the case of chiral compounds. At last, non aqueous Capillary Electrophoresis appears as a choice technique for the study of interactions in solution.
\end{abstract}

liquide. Si les grosses biomolécules sont compatibles uniquement avec les tampons aqueux et ne peuvent être étudiées que dans ce type de milieu, les composés hydrophobes, tels que certaines petites molécules d'intérêt pharmaceutique, méritent d'être séparés en milieu non aqueux, plus favorable à l'optimisation de la sélectivité.

En solution les molécules échantillons étant solvatées, le remplacement des molécules d'eau par des molécules de solvants organiques a un impact certain sur le comportement du soluté. Aussi considérera-t-on en premier lieu les caractéristiques des solvants organiques susceptibles d'influer sur le comportement électrophorétique des molécules échantillons, sans oublier celles pouvant modifier les équilibres chimiques et notamment acido-basiques. Le recours à l'ajout de solvant organique à une veine liquide aqueuse sera ensuite envisagé avant de considérer le développement de l'électrophorèse capillaire en milieu purement organique.

\section{Caractéristiques requises pour un solvant en électrophorèse capillaire}

L'électrophorèse capillaire a généré un intérêt considérable ces dernières années du fait qu'elle fait appel à une instrumentation relativement simple pour obtenir des séparations rapides et très efficaces, en consommant un minimum d'échantillon. Elle s'adapte aux composés ioniques et non ioniques, qu'ils soient de nature organique ou inorganique, quelle que soit leur masse moléculaire. Enfin elle se satisfait d'une préparation limitée de l'échantillon et peut séparer des composés présents à faible concentration.

L'électrophorèse capillaire, tout comme l'électrophorèse, a d'abord été mise en œuvre en milieu aqueux. Alors que l'usage des milieux non aqueux en électrophorèse remonte à plus d'une vingtaine d'années [1], ce n'est qu'en 1984 que Jorgenson et al. [2] ont montré, en effectuant une séparation de quinolines en milieu totalement anhydre, que cette technique apparaissait prometteuse pour les séparations en électrophorèse capillaire. Par la suite les travaux se sont faits plus nombreux, notamment depuis 1993, où le sujet a suscité un intérêt particulier parmi les analyticiens [3-7].

Pour réguler le flux électroosmotique et moduler la sélectivité, l'électrophorèse capillaire est conduite le plus souvent avec des mélanges tampon aqueux/solvant organique plutôt qu'en milieu organique pur. La plupart des études effectuées en milieu hydroorganique recourt généralement à un faible pourcentage $(<20 \%)$ de solvant organique dans la veine

\section{Courant, efficacité et temps d'analyse}

Les analyses effectuées par électrophorèse capillaire dépendent des mobilités électrophorétiques des substrats ainsi que du flux électroosmotique généré dans le milieu. Comme le montrent les relations (1) et (2) les mobilités électrophorétiques et électroosmotiques sont déterminées par la constante diélectrique $(\varepsilon)$ et la viscosité $(\eta)$ du milieu :

$$
\mu_{\mathrm{ep}}=\frac{2 \varepsilon \cdot \xi_{\text {ion }}}{3 \eta} \quad \text { (1) } \quad \mu_{\text {eo }}=\frac{\varepsilon \cdot \xi_{\text {cap }}}{\eta}
$$

où $\xi_{\text {ion }}$ et $\xi_{\text {cap }}$ sont respectivement les potentiels zeta de l'analyte et de la paroi du capillaire. A condition que les potentiels zeta soient égaux, plus le rapport $\varepsilon / \eta$ est grand pour un électrolyte donné, plus la migration et le flux électroosmotique sont élevés.

Les valeurs de $\varepsilon$ et $\eta$ ont été rassemblées dans le tableau I pour une dizaine de solvants, au côté d'autres paramètres physico-chimiques qui seront discutés ultérieurement. Le rapport $\varepsilon / \eta$ varie de 22,7 pour l'éthanol à 110,3 pour l'acétonitrile et le $N$-méthylformamide, laissant supposer des migrations variables et pour certains solvants plus rapides que dans l'eau. Effectivement la séparation du propranolol 
et de la felodipine opérée dans le $N$-méthylformamide nécessite seulement 35 secondes [9].

Pour ce qui est des mélanges binaires eau/solvant organique, $\eta$ varie de façon relativement complexe en fonction de la teneur en solvant organique [10]. Pour un certain nombre de solvants le rapport $\varepsilon / \eta$ diminue puis augmente. À titre d'exemple, dans le cas du méthanol, cette brusque modification de comportement est observée pour des teneurs supérieures à $60 \%$ en méthanol. Si l'on ajoute à cela que les solvants organiques peuvent affecter de façon complexe le potentiel zeta, on conçoit la difficulté à évaluer avec précision la résultante de tous ces effets.

Sauf cas particulier, le milieu électrophorétique est constitué d'un solvant, éventuellement d'un cosolvant et d'un ou plusieurs sels permettant de controler la force ionique et éventuellement le $\mathrm{pH}$. À concentration en sels égale, les courants généralement enregistrés dans les solvants organiques sont plus faibles que dans l'eau du fait de l'ionisation et de la dissociation des électrolytes plus réduites en milieu non aqueux. Ceci a pour conséquence de minimiser l'effet Joule correspondant à un dégagement de chaleur, qui peut être exprimé sous forme de puissance dissipée par unité de longueur par la relation :

$$
\frac{P}{L}=\frac{i^{2}}{\kappa \cdot \mathrm{A}}
$$

où $i$ est l'intensité de courant, $\mathrm{k}$ représente la conductance spécifique et $A$ la surface de la section du capillaire.

Il est alors possible :

- d'utiliser des capillaires de plus grand diamètre, admettant une charge échantillon supérieure et par suite une limite de détection plus grande;
- de maintenir la concentration en sel faible pour obtenir des courants de l'ordre du microampère, particulièrement compatibles avec le couplage électrophorèse capillaire/ spectrométrie de masse (CE/MS), car comparables à ceux générés au niveau de l'interface électrospray ionisation (ESI) ;

- de recourir à des champs plus élevés pour augmenter l'efficacité. En supposant que la seule source d'élargissement des pics est la diffusion, Jansson et Roeraade [9] ont décrit l'efficacité par unité de temps par la relation suivante :

$$
\frac{N}{t} \propto \frac{r}{k \cdot T \cdot \eta}\left(2 \xi_{\text {ion }}-3 \xi_{\text {cap }}\right)^{2}\left(\varepsilon_{0} \cdot \varepsilon_{\mathrm{r}} \cdot E\right)^{2}
$$

où $r=$ rayon de Stokes du substrat,

$k=$ constante de Boltzman,

$T=$ température,

$\eta=$ viscosité du solvant,

$\xi_{\text {ion }}$ et $\xi_{\text {cap }}$ potentiel zeta de l'ion et de la surface du capillaire,

$\varepsilon_{\mathrm{o}}=$ permittivité du vide,

$\varepsilon_{\mathrm{r}}=$ constante diélectrique relative du solvant,

$E=$ intensité du champ électrique.

Cette équation montre que les solvants présentant un rapport $\varepsilon^{2} / \eta$ élevé doivent conduire à des efficacités très grandes. C'est le cas notamment du $N$-méthylformamide pour lequel le rapport $\varepsilon^{2} / \eta$ atteint la valeur élevée de 20075 (cf. Tab. I), si on la compare à celle de l'eau qui n'est que de 6924. Des nombres de plateaux théoriques par mètre supérieurs à 500000 ont effectivement été observés dans ce solvant avec les acides aminés [11].

Plutôt que de recourir à des différences de potentiel plus élevées pour augmenter l'efficacité, on peut aussi envisager

\begin{tabular}{|c|c|c|c|c|c|c|c|}
\hline Solvant & $\eta(c P)$ à $25{ }^{\circ} \mathrm{C}$ & $\varepsilon$ & $\varepsilon / \eta$ & $\varepsilon^{2} / \eta$ & $p K$ & $\lambda_{\text {(cut off) }}(n m)$ & $T_{\text {éb }}\left({ }^{\circ} \mathrm{C}\right)$ \\
\hline eau & 0,89 & 78,5 & 88,2 & 6924 & 14,0 & $<190$ & 100 \\
\hline méthanol & 0,55 & 32,6 & 59,3 & 1932 & 17,2 & 205 & 64,6 \\
\hline éthanol & 1,07 & 24,3 & 22,7 & 552 & 18,9 & 210 & 78,5 \\
\hline $\mathrm{F}$ & 3,30 & 111 & 33,6 & 3734 & 16,8 & & 210 \\
\hline NMF & 1,65 & 182 & 110,3 & 20075 & 10,7 & & 182 \\
\hline DMF & 0,80 & 36,7 & 45,9 & 1684 & 29,4 & 268 & 153 \\
\hline DMA & 0,78 & 37,8 & 48,5 & 1832 & 24,0 & 268 & 166 \\
\hline DMSO & 2,00 & 46,7 & 23,3 & 1090 & 33,3 & 286 & 189 \\
\hline Acétone & 0,30 & 20,7 & 69,0 & 1428 & & 330 & 56,1 \\
\hline $\mathrm{ACN}$ & 0,34 & 37,5 & 110,3 & 4136 & * & 190 & 81,6 \\
\hline
\end{tabular}

Tableau I. Paramètres physico-chimiques de solvants susceptibles d'être utilisés en électrophorèse capillaire [8].

* Autoprotolyse non détectable. Abréviations : F : formamide, NMF : N-méthylformamide, DMF : diméthylformamide, DMA : diméthylacétamide, DMSO : diméthylsulfoxyde, ACN : acétonitrile. 
une réduction des temps d'analyse à l'aide d'un capillaire de longueur plus réduite.

\section{Sélectivité}

Dans la pratique, il est très souvent fait appel aux milieux non aqueux ou hydroorganiques pour modifier la sélectivité. En électrophorèse capillaire de zone, la sélectivité est basée sur des différences au niveau du rapport charge/rayon hydrodynamique du soluté. Aussi le type de solvant choisi est susceptible d'affecter l'un ou l'autre de ces paramètres voire les deux simultanément. Toutefois les solvants présentant des constantes diélectriques très faibles, tels que le dioxane, le chloroforme, l'acétate d'éthyle, le tétrahydrofuranne ou le chlorure de méthylène, qui ne sont pas favorables à la solubilisation d'électrolytes, ont été délaissés au profit de solvants dont la constante diélectrique est supérieure à 20 (cf. Tab. I).

Dans ce contexte, les propriétés acide-base de l'analyte peuvent varier de façon notable selon le milieu. Ainsi les solvants amphiprotiques à caractère acide renforcent la basicité des solutés tandis que ceux à caractère basique opèrent de façon inverse [12].

De même, les constantes d'association avec des espèces autres que les protons sont également susceptibles de varier d'un solvant organique à l'autre et d'être différentes de ce qu'elles sont dans l'eau. Ceci est valable notamment pour les constantes d'association des solutés avec un sélecteur chiral. Dans ces conditions, l'usage de solvants non aqueux doit permettre une meilleure flexibilité dans la méthode de développement et d'optimisation. À titre d'exemple [11], les énantiomères de la leucine dansylée sont bien séparés dans le formamide en présence de $\beta$-cyclodextrine $(10 \mathrm{mM})$ et de chlorure de sodium $(10 \mathrm{mM})$, mais présentent des temps d'analyse assez importants, respectivement de 8,5 et 9,5 minutes environ. Dans les mêmes conditions, les temps sont ramenés à 2 minutes dans le $N$-méthylformamide, mais avec une résolution nulle. Toutefois en augmentant la concentration en $\beta$-cyclodextrine de 10 à $60 \mathrm{mM}$, on obtient alors une résolution satisfaisante.

\section{Détection}

Il existe plusieurs types de détecteur en électrophorèse capillaire, mais le mode le plus répandu demeure la détection UV directe. Généralement les solvants organiques absorbent plus dans le domaine UV que ne le fait l'eau (cf. Tab. I), ce qui constitue un désavantage certain. C'est d'ailleurs une des raisons pour lesquelles le méthanol et l'acétonitrile sont les solvants les plus utilisés en milieu non aqueux. Il est cependant à signaler que d'autres modes de détection peuvent être envisagés, la détection UV indirecte [13,14], la détection fluorimétrique indirecte induite par LASER [15,16], la détection électrochimique qui est bien adaptée aux solvants dipolaires aprotiques comme le dimethylformamide, le dimethylsulfoxyde et l'acétonitrile [17-19] ou encore le couplage CE/MS qui nécessite des solvants volatils tels que le méthanol ou l'acétonitrile [4,20-22]. Ainsi dans l'étude du métabolisme du tamoxifène en électrophorèse capillaire cou- plée à la spectrométrie de masse (ESI), il a été constaté une meilleure résolution et une sensibilité plus grande en milieu organique par rapport au milieu aqueux [22].

\section{Solubilité}

Les solvants organiques sont aptes, à condition de présenter une constante diélectrique suffisamment élevée, à dissoudre un grand nombre de sels servant d'électrolyte et assurant un courant électrique stable.

Au niveau des solutés, un certain nombre de composés peu solubles dans l'eau, telles les substances hydrophobes, mérite d'être analysé en milieu organique [21,23]. Ainsi il a été signalé que la $\mathrm{H}_{2}$-mifentidine antagoniste [4] présente une meilleure solubilité et une tendance moindre à s'adsorber sur les parois du capillaire en milieu non aqueux.

Les solvants organiques sont par ailleurs propices à la solubilisation d'additifs facilitant notamment la séparation de composés chiraux. Ainsi la quinine a été utilisée dans le méthanol comme sélecteur chiral pour séparer les acides aminés énantiomères dérivatisés par le chlorure de 3,5-dinitrobenzoyle [24]. Par ailleurs des acides aminés dansylés ont été séparés aisément dans le $N$-méthylformamide à l'aide de $\beta$-cyclodextrine comme sélecteur chiral, qui s'avère 35 fois plus soluble en milieu organique qu'en milieu aqueux [25].

\section{Autres considérations}

Le point d'ébullition des solvants est également un paramètre à considérer (cf. Tab. I), notamment en fonction du type de détection. Ainsi les solvants plus volatils que l'eau présentent un intérêt pour la détection en spectrométrie de masse. Mais ces mêmes solvants sont plus difficiles à manipuler du fait de leur volatilité entraînant une certaine évaporation. Celle-ci peut se manifester par un manque de reproductibilité ou encore par des ruptures du courant. Au contraire, les solvants moins volatils que l'eau présenteront les avantages et les inconvénients inverses.

Il faut également tenir compte d'un certain nombre de critères tels que la purification plus ou moins aisée, le coût ou encore la toxicité du solvant.

Pour ces différentes raisons de facilité de mise en oeuvre, le méthanol et l'acétonitrile sont devenus les solvants les plus utilisés en électrophorèse capillaire non aqueuse.

Ayant passé en revue les caractéristiques à prendre en compte avant de choisir, comme veine liquide en électrophorèse capillaire, un solvant organique qui réponde le mieux possible aux exigences liées au système de détection, aux problèmes de solubilité et qui permette l'obtention d'une résolution satisfaisante pour les constituants du mélange qu'on se propose d'étudier, nous allons considérer maintenant un certain nombre de séparations effectuées en milieu hydroorganique ou totalement organique. La liste des travaux rapportés n'a pas la prétention d'être exhaustive, le but recherché étant simplement d'apporter une vision relativement complète des domaines dans lesquels une telle approche mérite d'être envisagée. 


\section{Analyses effectuées en milieu non aqueux par électrophorèse capillaire}

Un grand nombre de séparations a déjà été effectué avec succès par électrophorèse capillaire, soit en milieu hydroorganique, soit en milieu totalement anhydre comprenant un ou plusieurs solvants organiques en mélange. Les analyses référencées dans le tableau II rapportent des séparations où le solvant organique tient lieu de co-solvant ajouté à la veine liquide aqueuse, tandis que dans le tableau III sont indiquées des analyses pratiquées en milieu purement organique.

Pour les séparations en milieu hydroorganique, il est à noter que les teneurs en co-solvant sont généralement inférieures à $30 \%$ et n'atteignent qu'exceptionnellement des pourcentages élevés. Comme l'indique le tableau II, les

Tableau II. Séparations effectuées par électrophorèse capillaire en milieu hydroorganique.

\begin{tabular}{|c|c|c|c|}
\hline Composés & Co-Solvants organiques & Particularité & Réfs. \\
\hline $\begin{array}{l}\text { isoquinoline } \\
\text { lysozyme }\end{array}$ & ACN & & $2(1984)$ \\
\hline molécules organiques neutres & ACN & $\begin{array}{l}\text { association solvatophobe avec des } \\
\text { ions tétraalkylammonium }\end{array}$ & $26(1986)$ \\
\hline amines & 2-Propanol & gradient d'élution en MEKC & $27(1988)$ \\
\hline $\begin{array}{l}\text { marqueurs de flux (umbelliferone, } \\
\text { riboflavine, 4-nitroaniline) }\end{array}$ & $\begin{array}{l}\mathrm{MeOH}, \mathrm{EtOH}, 2 \text {-propanol, } \\
\mathrm{ACN} \text {, acétone, DMSO }\end{array}$ & étude du flux électroosmotique & $10(1991)$ \\
\hline $\begin{array}{l}\beta \text { bloquants } \\
\text { (adrénergiques) }\end{array}$ & $\begin{array}{l}\text { acétone, ACN, 2-propanol, } \\
\text { éthylèneglycol, MeOH EtOH }\end{array}$ & MEKC & $28(1993)$ \\
\hline $\begin{array}{l}\text { quinoline, isoquinoline, acide } \\
\text { p-aminobenzoïque, tryptophane, } \\
\text { dipeptides }\end{array}$ & $\begin{array}{c}\text { MeOH, EtOH, propanol, } \\
\text { ACN, DMF }\end{array}$ & & $29(1993)$ \\
\hline $\begin{array}{l}\text { métabolisme de médicaments } \\
\text { halopéridol }\end{array}$ & $\mathrm{MeOH}$ & & $30(1993)$ \\
\hline protéines & 2-propanol & CE/MS & $23(1994)$ \\
\hline peptides & $\mathrm{F}$ & & $5(1994)$ \\
\hline anions inorganiques & $\mathrm{MeOH}, \mathrm{DMF}$ & détection ampérométrique et UV indirecte & $19(1995)$ \\
\hline $\begin{array}{l}n \text {-alcane sulfonates } \\
\text { acides carboxyliques }\end{array}$ & $\begin{array}{c}\text { ACN, EtOH, 2-propanol } \\
\text { EtOH }\end{array}$ & $\begin{array}{l}\text { détection fluorimétrique } \\
\text { indirecte induite par laser }\end{array}$ & $16(1995)$ \\
\hline alkylbenzènes, tensioactifs & ACN, 2-propanol & SDS et forte concentration en cosolvant & $31(1995)$ \\
\hline $\begin{array}{l}\text { benzène, acétonehydrocarbures } \\
\text { polycycliques aromatiques }\end{array}$ & $\mathrm{ACN}, \mathrm{MeOH}, \mathrm{F}, \mathrm{DMF}, \mathrm{DMSO}$ & $\begin{array}{l}\text { étude du flux électroosmotique } \\
\text { électrochromatographie }\end{array}$ & $32(1997)$ \\
\hline acides gras & $\mathrm{MeOH}$ & MEKC en présence de BRIJ 35 & $33(1997)$ \\
\hline alcools gras éthoxylés & $\mathrm{ACN}$ & & $34(1998)$ \\
\hline
\end{tabular}

Abréviations des solvants identiques à celles du tableau I. MEKC : chromatographie micellaire électrocinétique. CE/MS : électrophorèse capillaire/spectrométrie de masse. 
Tableau III. Séparations effectuées par électrophorèse capillaire en milieu organique.

\begin{tabular}{|c|c|c|c|}
\hline Composés & Solvants & Particularité & Réfs. \\
\hline pyrazoloacridine ; métabolisme & $\mathrm{MeOH}$ & isotachophorèse préconcentration & $36(1993)$ \\
\hline métabolites & $\mathrm{MeOH}$ & $1^{\text {er }}$ couplage CE/MS & $37(1994)$ \\
\hline $\mathrm{H}_{2}$-mifentidine antagoniste & $\mathrm{MeOH}$ & CE/MS & $4(1994)$ \\
\hline $\mathrm{F}, \mathrm{NMF}$ & $=$, DMF, DMA, DMSO, ACN, & , $\mathrm{MeOH}$ & 7 (1995) \\
\hline polyéthers & $\mathrm{MeOH}$ & détermination de constantes de complexation & $35(1995)$ \\
\hline acides aliphatiques et aromatiques & $\mathrm{MeOH}$ & & $13(1995)$ \\
\hline $\begin{array}{l}\text { acides carboxyliques et produits } \\
\text { pharmaceutiques }\end{array}$ & NMF & & $9(1995)$ \\
\hline $\begin{array}{l}\text { médicaments et métabolites } \\
\text { halopéridol, mifentidine }\end{array}$ & $\mathrm{MeOH}$ & CE/MS & $21(1995)$ \\
\hline tamoxifène et métabolites & $\mathrm{MeOH}$ & CE/MS (electrospray) tensioactifs comme additifs & $22(1996)$ \\
\hline tetracycline & $\mathrm{MeOH} / \mathrm{ACN}+\mathrm{DMF}$ & & $38(1996)$ \\
\hline $\begin{array}{l}\text { camphorsulphonates, } \\
\text { médicaments chiraux }\end{array}$ & ACN & Electrophorèse capillaire chirale & 39 (1996) \\
\hline acides aminés dansylés & $F, N M F$ & $\begin{array}{l}\quad \beta \text {-cyclodextrines ; } \\
\text { Electrophorèse capillaire chirale }\end{array}$ & $11(1996)$ \\
\hline $\begin{array}{l}\text { racémiques de produits } \\
\text { pharmaceutiques, amines }\end{array}$ & F, NMF, DMF & $\begin{array}{l}\beta \text {-et } \gamma \text {-cyclodextrines } \\
\text { Electrophorèse capillaire chirale }\end{array}$ & $40(1996)$ \\
\hline tensioactifs & $\mathrm{MeOH}, \mathrm{MeOH} / \mathrm{ACN}$ & & $14(1996)$ \\
\hline cations alcalins et alcalino-terreux & $\mathrm{MeOH}, \mathrm{ACN}$ & & $41(1996)$ \\
\hline médicaments & $\mathrm{ACN} / \mathrm{MeOH} / \mathrm{CH}_{3} \mathrm{COOH}$ & & $42(1996)$ \\
\hline $\begin{array}{l}\text { amines dérivées de l'imipramine } \\
\text { amines, acides }\end{array}$ & $\begin{array}{l}\text { MeOH, ACN, NMF, DMF, } \\
\text { DMA, DMSO, ACN }\end{array}$ & effet de traces d'eau & 43 (1996) \\
\hline $\begin{array}{l}\text { acides aromatiques, acides aminés, } \\
\text { amino-alcools chiraux }\end{array}$ & $\mathrm{F}$ & $\begin{array}{l}\text { éther couronne comme sélecteur chiral, } \\
\text { Electrophorèse capillaire chirale }\end{array}$ & $44(1997)$ \\
\hline $\begin{array}{l}\text { acides, médicaments, colorants, } \\
\text { tensioactifs }\end{array}$ & $\mathrm{MeOH} / \mathrm{ACN}$ & $\begin{array}{c}\text { parfois } \beta \text {-cyclodextrines, } \\
\text { Electrophorese capillaire chirale }\end{array}$ & $45(1997)$ \\
\hline hydrocarbures polyaromatiques & $\mathrm{ACN}$ & interactions de type donneur/accepteur & $46(1997)$ \\
\hline chlorure de chlorprothixène & $\mathrm{MeOH} / \mathrm{ACN}$ & $\begin{array}{l}\text { séparation d'isomères } \mathrm{Z} / \mathrm{E} \text {, } \\
\text { de diastéréoisomères cationiques }\end{array}$ & $47(1997)$ \\
\hline acides carboxyliques, phénols, alcools & ACN & $\begin{array}{c}\text { détermination de constantes de formation } \\
\text { d'anions hétéroconjugués }\end{array}$ & $48(1997)$ \\
\hline $\begin{array}{l}\text { polyéthers, polymères, phénols, } \\
\text { éthers couronne }\end{array}$ & ACN & & 49 (1998) \\
\hline cimétidine, produits pharmaceutiques & $\mathrm{ACN} / \mathrm{MeOH}+\mathrm{CH}_{3} \mathrm{COOH}$ & & $50(1998)$ \\
\hline ions métalliques & $\begin{array}{c}\mathrm{EtOH} / \mathrm{DMF} \\
\mathrm{EtOH} / \mathrm{ACN} / \mathrm{CH}_{3} \mathrm{COOH}\end{array}$ & Electrophorèse capillaire semi-préparative & $\begin{array}{l}51(1998) \\
52(1998)\end{array}$ \\
\hline $\begin{array}{l}\text { médicaments anti-inflammatoires, } \\
\text { acides aminés, profènes }\end{array}$ & $\mathrm{F}, \mathrm{NMF}, \mathrm{MeOH}, \mathrm{DMSO}$ & Electrophorèse capillaire chirale & $53(1998)$ \\
\hline anions benzoates & ACN + polyéthylèneglycol & étude des liaisons hydrogène & $54(1998)$ \\
\hline sulfonamides & $A C N$ & Complexation avec $\mathrm{Ag}(\mathrm{I})$ & 55 (1998) \\
\hline
\end{tabular}

Abréviations des solvants identiques à celles du tableau I. 
composés étudiés sont de nature très variée. L'ajout de solvant organique permet d'étudier des protéines hydrophobes [23], tout en se prêtant bien à une détection par spectrométrie de masse, qui favorise la caractérisation structurale. La présence de co-solvant organique dans le cas d'alcools gras comportant une chaine éthoxylée [34] conduit à l'établissement d'un électrophorégramme constituant une véritable empreinte, directement utilisable pour des comparaisons entre produits commerciaux. De plus il apparaît que les milieux hydroorganiques sont particulièrement favorables à l'étude des médicaments et de leurs métabolites [30]. Dans certains cas, il a été signalé une amélioration au niveau de la résolution [28,31] ou encore de l'efficacité [19]. Mais l'intérêt essentiel d'introduire un co-solvant organique dans la veine liquide en électrophorèse capillaire est d'engendrer une modification de la sélectivité favorable à la séparation des constituants d'un mélange [19], qui se traduit même parfois par une modification de l'ordre d'élution [29].

L'utilisation en électrophorèse capillaire d'une veine liquide purement organique est apparue plus tardivement, mais constitue une solution à laquelle les analystes recourent de plus en plus, comme en témoigne le nombre grandissant de publications parues au cours des dix dernières années.

L'électrophorèse capillaire en milieu non aqueux se prête particulièrement bien à l'étude du devenir des médicaments et de leurs métabolites, comme l'attestent différents travaux $[21,22,36,37,38,42,50]$. L'emploi de solvants organiques s'est par ailleurs révélé très profitable pour la séparation de molécules chirales, comme en témoigne un nombre d'études déjà important [11,39,40,44,45,47,53]. En effet les solvants organiques qui présentent une meilleure solubilité pour les sélecteurs chiraux que l'eau, permettent de profiter d'une gamme de concentration en additif plus étendue. Des grosses molécules, tensioactifs [14], polymères divers [49] ont été analysées en électrophorèse capillaire dans le méthanol et l'acétonitrile avec succès.

Étant donné que les solvants organiques renferment obligatoirement de l'eau à l'état de traces difficilement contrôlables, il est intéressant de noter que l'ajout à l'acétonitrile d'eau, jusqu'à $1 \%$, n'entraîne que peu, voire pas de changement de sélectivité, pour des solutés cationiques comme anioniques [43].

Enfin le recours à l'électrophorèse capillaire en milieu purement organique constitue une façon d'aborder la chimie des solutions. Il permet notamment l'étude d'interactions faibles comme la liaison hydrogène [54] et de façon quantitative la détermination de constantes de complexation, par exemple entre polyéthers et cations [35], ou entre donneurs et accepteurs [46] ou encore la mesure de constantes de formation d'anions hétéroconjugués [48].

\section{Conclusion}

L'électrophorèse capillaire en milieu non aqueux s'affirme comme une technique intéressante pour augmenter l'effica- cité, réduire l'adsorption, résoudre des problèmes liés à la solubilité. Mais son côté le plus attractif réside dans la facilité qu'elle offre de faire varier la sélectivité en changeant soit la nature du solvant, soit la nature et la concentration de l'électrolyte. En effet en faisant appel à des propriétés physico-chimiques différentes (liaison hydrogène, caractère donneur/accepteur...), on est en droit d'attendre des changements de sélectivité très importants.

Aussi cette technique s'annonce prometteuse pour suivre le devenir des médicaments et de leurs métabolites dans les fluides biologiques, après concentration par extraction solide et élution à l'aide de solvants organiques. Elle l'est également en électrophorèse capillaire chirale, en permettant l'utilisation d'un plus grand nombre d'additifs, dans une gamme de concentration plus étendue. Elle se prête aussi au couplage avec la spectrométrie de masse, qui constitue la solution d'avenir pour identifier les solutés et abaisser les seuils de détection.

À côté de cet aspect analytique, l'électrophorèse capillaire en milieu non aqueux doit contribuer à une meilleure connaissance des interactions ions/ions et ions/solvants et permettre la détermination de grandeurs physiques (constantes d'équilibre...). Des études sont nécessaires pour mieux appréhender et mieux comprendre le comportement de différents systèmes électrolyte/solvant. Une méthode de développement plus rationnelle pourra alors être établie pour résoudre la séparation d'espèces proches.

En conclusion, bien contrôlée l'électrophorèse capillaire en milieu non aqueux, qui a déjà son sigle en anglais « NACE », offre une alternative intéressante aux méthodes de l'électrophorèse capillaire traditionnelle pour la séparation d'un grand nombre d'espèces, notamment à caractère hydrophobe.

\section{Références}

1. Korchemnaya, E. K.; Ermakov, A. N.; Bochkova, P. L. J. Anal. Chem. USSR, (Engl. Transl.) 1978, 33, 635-639.

2. Walbroehl, Y.; Jorgenson, J. W. J. Chromatogr. 1984, 315, 135143.

3. Tomlinson, A. J.; Benson, L. M.; Naylor, S. $L C-G C$ 1994, 12, 122-130.

4. Tomlinson, A. J.; Benson, L. M.; Gorrod, J. W.; Naylor, S. J. Chromatogr. B 1994, 657, 373-381.

5. Sahota, R. S.; Khaledi, M. G. Anal. Chem. 1994, 66, 11411146.

6. Ng, C. L.; Lee, H. K.; Li, S. F. Y. J. Liq. Chromatogr. 1994, 17, 3837-3857.

7. Bjornsdottir, I.; Hanssen, S. H. J. Chromatogr. A 1995, 711, 313-322.

8. Riddick, J. A.; Bunger, W. B.; Organic solvents. Physical properties and methods of purification; Wiley-Interscience, New York, 1970.

9. Jansson, M.; Roeraade, J. Chromatographia 1995, 40, 163169.

10. Schwer, C.; Kenndler, E. Anal. Chem. 1991, 63, 1801-1807.

11. Valko, I. E.; Siren, H.; Riekkola, M. L.; Chromatographia 1996, 43, 242-246. 
12. Skoog, D. A.; West, D. M.; Fundamentals of Analytical Chemistry; CBS College Publishing, Philadelphia, 1982, p. 265.

13. Chiari, M.; Kenndler, E. J. Chromatogr. A 1995, 716, 303-309.

14. Salimi-Moosavi, H.; Cassidy, R. M. Anal. Chem. 1996, 68, 293-299.

15. Kuhr, W. G.; Yeung, E. S. Anal. Chem. 1988, 60,1832-1836.

16. Desbène, A. M.; Morin, C. J.; Mofaddel, N. L.; Groult, R. S. J. Chromatogr. A 1995, 716, 279-290.

17. Wallingford, R. A.; Ewing, A. G. Anal. Chem. 1989, 61, 98100 .

18. Sloss, S.; Ewing, A. G. Anal. Chem. 1993, 65, 577-581.

19. Salimi-Moosavi, H.; Cassidy, R. M. Anal. Chem. 1995, 67, 1067-1073.

20. Naylor, S.; Tomlinson, A. J.; Beson, L. M.; Gorrod, J. W. Eur. J. Drug. Metab. Pharmacokinet. 1994, 19, 235-240.

21. Tomlinson, A. J.; Benson, L. M.; Naylor, S. LC-GC Int. 1995, $8,210-216$

22. Lu, W.; Poon, G. K.; Carmichael, P. L.; Cole, R. B. Anal. Chem. 1996, 68, 668-674.

23. Weinmann, W.; Maier, C.; Baumeister, K.; Przybylski, M.; Parker, C. E.; Tomer, K. B. J. Chromatogr. A 1994, 664, 271275.

24. Stalcup, A. M.; Gham, K. H. J. Microcol. Sep. 1996, 8, 145150.

25. Valko, I. E.; Siren, H.; Riekkola, M. L. J. Chromatogr. A 1996, 737, 263-272.

26. Walbroehl, Y.; Jorgenson, J. W. Anal. Chem. 1986, 58, 479481.

27. Balchunas, A. T.; Sepaniak, M. J. Anal. Chem. 1988, 60, 617621.

28. Lukkari, P.; Vuorela, H.; Riekkola, M. L. J. Chromatogr. A 1993, 655, 317-324

29. Janini, G. M.; Chan, K. C.; Barnes, J. A.; Muschik, G. M.; Issaq, H. J. Chromatographia 1993, 35, 497-502.

30. Tomlinson, A. J.; Benson, L. M.; Landers, J. P.; Scanlan, G. F.; Fang, J.; Gorrod, J. W.; Naylor, S. J. Chromatogr. A 1993, 652, 417-426.

31. Desbène, P. L.; Rony, C. M. J. Chromatogr. A 1995, 689, 107121.

32. Wright, P. B.; Lister, A. S.; Dorsey, J. G. Anal. Chem. 1997, $69,3251-3259$
33. Collet, J.; Gareil, P. J. Chromatogr. A 1997, 792, 165-177.

34. Heinig, K.; Vogt, C.; Werner, G. Anal. Chem. 1998, 70, 18851892.

35. Okada, T. J. Chromatogr. A 1995, 695, 309-317.

36. Benson, L. M.; Tomlinson, A. J.; Reid, J. M.; Walker, D. L.; Ames, M. M.; Naylor, S. J. High Resolut. Chromatogr. 1993, $16,324-326$

37. Tomlinson, A. J.; Benson, L. M.; Naylor, S. J. High Resolut. Chromatogr. 1994, 17, 175-177.

38. Tjornelund, J.; Hansen, S. H. J. Chromatogr. A 1996, 737, 291300

39. Bjornsdottir, I.; Hansen, S. H., Terabe, S. J. Chromatogr. A 1996, 745, 37-44.

40. Wang, F.; Khaledi, M. G. Anal. Chem. 1996, 68, 3460-3467.

41. Salimi-Moosavi, H.; Cassidy, R. M. J. Chromatogr. A 1996, 749, 279-286.

42. Leung, G. N. W.; Tang, H. P. O.; Tso, T. S. C.; Wan, T. S. M. J. Chromatogr. A 1996, 738, 141-154.

43. Hansen, S. T.; Tjornelund, J.; Bjornsdottir, I. Trends Anal. Chem. 1996, 15, 175-180.

44. Mori, Y.; Ueno, K.; Umeda, T. J. Chromatogr. A 1997, 757, 328-332.

45. Altria, K. D.; Bryant, S. M. Chromatographia 1997, 46, 122130

46. Miller, J. L.; Khaledi, M. G.; Shea, D. Anal. Chem. 1997, 69, 1223-1229.

47. Hansen, S. H.; Bjornsdottir, I.; Tjornelund, J. J. Chromatogr. A 1997, 792, 49-55.

48. Okada, T. J. Chromatogr. A 1997, 771, 275-284.

49. Okada, T. J. Chromatogr. A 1998, 804, 17-28.

50. Ellis, D. R.; Palmer, M. E.; Tetler, L. W.; Eckers, C. J. Chromatogr. A 1998, 808, 269-275.

51. Liu, B. F.; Liu, L. B.; Cheng, J. K. Chemical J. Chinese Univ. 1998, 19, 1232-1235.

52. Valko, I. E.; Porras, S. P.; Riekkola, M. L. J. Chromatogr 1998, 813, 179-186.

53. Wang, F.; Khaledi, M. G.; J. Chromatogr. 1998, 817, 121-128.

54. Esaka, Y.; Yoshimura, K.; Goto, M.; Kano, K. J. Chromatogr. 1998, 822, 107-115.

55. Wright, P.B.; Dorsey, J.G. J. High. Resolut. Chromatogr. 1998, $21,498-504$. 